\title{
Performance Improvement in a Fingerprint Classification System Using Anisotropic Diffusion
}

\author{
Gonzalo Vallarino, Gustavo Gianarelli, Jose Barattini, Alvaro Gómez, \\ Alicia Fernández, and Alvaro Pardo \\ Institute of Electrical Engineering \\ Faculty of Engineering \\ Universidad de la República \\ Montevideo, Uruguay
}

\begin{abstract}
In a previous work, [1], we evaluated a classification algorithm based on the Karu-Jain method [2] and compared the performance with a fully manual method used at the Dirección Nacional de Identificación Civil (DNIC). In this paper, we analyze the high performance improvement achieved using anisotropic diffusion instead of pure averaging for the directions smoothing. We also define a quality measure that shows high correlation with the experts' criteria. The results are evaluated over 2800 images extracted from a 4 million fingerprint card archive maintained by DNIC.
\end{abstract}

\section{Introduction}

This work is part of an ongoing collaboration between the Universidad de la República and the Dirección Nacional de Identificación Civil (DNIC) concerned with civil identification affairs in Uruguay. The goal of this joint project is to evaluate an automatic fingerprint classification system compatible with the manual method that has been used by DNIC for several years. DNIC's classification scheme is based on the Vucetich system that has four fundamental classes: Arch, Right and Left Loop, and Whorl. In [1] we presented the results obtained in a first stage of the project. In this work we analyze the improvements achieved using anisotropic diffusion for direction smoothing instead of mean filters. For classification we propose some modifications to the algorithm proposed by Karu and Jain [2]. This algorithm is based on a singularities approach. The classification uses some heuristic criteria applied on the number and position of singularities.

To evaluate the algorithm we use the database described in [1], which is a representative sample of more than 4 hundred individual fingerprint cards from the national archive held by DNIC. Each card has a ten-print image and the corresponding manual classification formula provided by human experts. Given the heterogeneity of fingerprints obtained from the database, it becomes mandatory to have a quality measure in order to reject bad impressions. 
We define a global quality measure that takes into account the whole fingerprint and a local quality measure that only uses the regions surrounding the singularities. To compute these measures we use Gabor Filters as described in [3]

The quality of a fingerprint image is estimated using global and local features and also the number of steps in the iterative smoothing process. Based on this quality measure the fingerprints are divided into three categories: good, poor and bad. We learn the decision thresholds using a training set categorized by the DNIC experts.

The paper is organized as follows: In section 2 we describe the fingerprint database. In section 3 we describe the anisotropic diffusion algorithm. In section 4 the classification algorithm. In section 5 we present the results and the performance of the algorithm tested on our database. In section 6 we present the quality measure. Finally, in section 7 we outline the conclusions of this work.

\section{Fingerprint Database}

DNIC holds the fingerprints of more than four million people. The fingerprints of each individual are stored in a paper card as shown in [1]. The card archive is indexed and physically ordered by the ten-fingerprint classification formula of each individual. In order to test the classification algorithm, the whole paper card archive was sampled and more than four hundred cards were digitized to obtain over 4000 fingerprint digital images. The cards were digitized at a resolution of $500 \mathrm{dpi}$ and the fingerprints extracted to become $512 \times 480$ pixels images. In [1] we showed some statistics on the sample fingerprint database.

\section{The Anisotropic Diffusion Algorithm}

The direction vector field of the fingerprint is one of the most important features used in classification algorithms. Following [2] we perform a smoothing of the directions before classification. Instead of using a simple mean filter, we propose to use anisotropic diffusion. One of the main drawbacks with the mean filter is that it rapidly destroys singularities, especially deltas. Furthermore, the number of mean filtering iterations needed to obtain a reasonable direction field depends on each fingerprint.

On the other hand, anisotropic diffusion, is well known to respect singularities better than mean filtering. Also, it is known that during the evolution of the anisotropic diffusion singularities can disappear but never be created [5].

Although we obtained good results with the anisotropic diffusion filter, we are aware that there exist other diffusion algorithms that deal directly with directions and avoid artificial normalization steps. For example, this approach of direction diffusion was introduced in [6], and a complete framework for direction diffusion was introduced in [8]. In [8] Tang, Sapiro and Caselles introduce the algorithms for orientation diffusion based on the harmonic maps theory. In this way, they guarantee that during the continuous evolution, the diffused orientation remain living on the unit circle. Although, the same is not valid for the 
corresponding numerical implementations, some numerical advances have been recently made. We leave the study of this for future research.

For a continuous image $p: \Omega\left(\subset R^{2}\right) \rightarrow R$ the anisotropic diffusion evolution is given by the following PDE:

$$
\frac{\partial p}{\partial t}=\nabla\left(\frac{\nabla p}{\sqrt{\beta^{2}+\|\nabla p\|^{2}}}\right)
$$

For discrete images, we use the following numerical implementation (see [4] for details on this point):

$$
\begin{aligned}
\frac{p_{j, k}^{t+\Delta t}-p_{j, k}^{t}}{\triangle t}= & D_{j}^{-}\left\{\frac{D_{j}^{+} p_{j, k}^{t}}{\sqrt{\beta^{2}+\left\|D_{k}^{+} p_{j, k}^{t}\right\|^{2}+\left\|m\left(D_{j}^{+} p_{j, k}^{t}, D_{j}^{-} p_{j, k}^{t}\right)\right\|^{2}}}\right\}+\ldots \\
& \ldots+D_{k}^{-}\left\{\frac{D_{j}^{+} p_{j, k}^{t}}{\sqrt{\beta^{2}+\left\|D_{k}^{+} p_{j, k}^{t}\right\|^{2}+\left\|m\left(D_{j}^{+} p_{j, k}^{t}, D_{j}^{-} p_{j, k}^{t}\right)\right\|^{2}}}\right\}
\end{aligned}
$$

where $D_{j}^{ \pm}$y $D_{k}^{ \pm}$are the forward and backward differences $j$ and $k$, and the operator $m($.$) is m(x, y)=\operatorname{minmod}(x, y)=\min (x, y)[\operatorname{sign}(|x|)+\operatorname{sign}(|y|)] / 2$.

The numerical stability condition is easily computed to be:

$$
\triangle t \leq \beta / 4
$$

\subsection{Implementation}

In the numerical implementation we use the following parameters: $\beta=0.01$ and $\Delta t=\beta / 4.5$.

The anisotropic diffusion filter is applied to the matrices $R_{\cos }=\cos (2 \Theta)$ and $R_{\text {sin }}=\sin (2 \Theta)$, where $\Theta$ is the direction angle. Since we are dealing with a direction that lives in the unit circle, after each iteration of the anisotropic diffusion filter we apply a normalization step. Finally the direction angle $\Theta(i, j)$ is obtained with: $\Theta(i, j)=0.5 * \operatorname{atan}\left(R_{\sin }(i, j) / R_{\cos }(i, j)\right)$.

For the parameters mentioned above we empirically found that 200 iterations are enough to correctly classify poor fingerprints which are the majority in our database. This may not be an ideal stopping criteria for good fingerprints, and bad fingerprints. In the case of bad fingerprints, we usually need more iterations.

\section{Classification Algorithm}

In this section we describe the classification algorithm used. We modified the algorithm proposed by Karu and Jain [2] for a four-class problem.

We now describe its main steps and the modifications we have introduced. The first step is the computation of a directional image, corresponding to ridge directions at each pixel in the input image. All possible directions are quantized to eight discrete values equally spaced around the unit circle. Directions are 
then converted to vector form (i.e. (x,y) coordinate pairs), giving an appropriate representation to perform the smoothing operations described in Section 3. The original image is averaged in $8 \times 8$ windows giving a reduced directional image of size $64 \times 60$. Singularities are located computing the Poincaré index at every pixel (in the reduced image) in a $2 \times 2$ neighbourhood. Doing this we label each pixel as either: Ordinary, Delta, or Core point.

Based on the number and location of Delta and Core points, the fingerprint is adequately classified. As unwanted spurious singularities may appear in the first step we use an iterative approach where the directional image is successively smoothed, until we can classify it. The main drawback of the algorithm was the influence of spurious singularities that cause classification errors. Unwanted singular points come from many sources: low quality image within the fingerprint area, spurious artifacts outside the fingerprint area: typed words, lines, etc.

As a first pre-processing step, we normalize the image and make a segmentation to remove spurious elements. In a second step, we make some checking procedures to eliminate false singularities. This classification into the four classes (Arch, Left and Right Loop and Whorl) is done based on the number and position of the detected Deltas and Cores.

\section{Results and Evaluation}

In tables 1 to 4 we show the results of the classification algorithm over 2800 fingerprints which includes good, poor and bad ones. As we can see, the anisotropic diffusion algorithm performs better than the mean filter smoothing.

\subsection{Test Platform}

The algorithms were coded in $\mathrm{C}++$ using the image processing library VIL which is part of VXL. The tests were conducted on an AMD Athlon $1300 \mathrm{MHz}$ with

Table 1. Mean filter performance for singularities classification

\begin{tabular}{|c|c|c|c|c|}
\hline \multicolumn{5}{|c|}{ Mean filter performance } \\
\hline & Arch & Left loop & Right loop & Whorl \\
\hline Arch & $73(71.57 \%)$ & $21(2.41 \%)$ & $12(1.57 \%)$ & $10(0.99 \%)$ \\
\hline Left loop & $7(6.86 \%)$ & $816(93.69 \%)$ & $20(2.62 \%)$ & $69(6.83 \%)$ \\
\hline Right loop & $21(20.59 \%)$ & $21(2.41 \%)$ & $706(92.41 \%)$ & $90(8.91 \%)$ \\
\hline Whorl & $1(0.98 \%)$ & $13(1.49 \%)$ & $26(3.40 \%)$ & $841(83.27 \%)$ \\
\hline Total & $102(100 \%)$ & $871(100 \%)$ & $764(100 \%)$ & $1010(100 \%)$ \\
\hline
\end{tabular}

Table 2. Mean filter classification performance

\begin{tabular}{|c|c|}
\hline \multicolumn{2}{|c|}{ Mean filter performance } \\
\hline Correct classification & $2436(87.00 \%)$ \\
\hline Wrong classification & $311(11.11 \%)$ \\
\hline Not classified & $53(1.89 \%)$ \\
\hline Total & $2800(100 \%)$ \\
\hline
\end{tabular}


Table 3. Anisotropic diffusion filter performance for singularities classification

\begin{tabular}{|c|c|c|c|c|}
\hline \multicolumn{5}{|c|}{ Anisotropic diffusion performance } \\
\hline & Arch & Left loop & Right loop & Whorl \\
\hline Arch & $108(84.38 \%)$ & $39(4.16 \%)$ & $8(0.94 \%)$ & $3(0.34 \%)$ \\
\hline Left loop & $9(7.03 \%)$ & $865(92.32 \%)$ & $15(1.75 \%)$ & $20(2.28 \%)$ \\
\hline Right loop & $11(8.59 \%)$ & $16(1.71 \%)$ & $811(94.85 \%)$ & $22(2.51 \%)$ \\
\hline Whorl & $0(0 \%)$ & $17(1.81 \%)$ & $21(2.46 \%)$ & $831(94.86 \%)$ \\
\hline Total & $128(100 \%)$ & $937(100 \%)$ & $855(100 \%)$ & $876(100 \%)$ \\
\hline
\end{tabular}

Table 4. Anisotropic diffusion filter classification performance

\begin{tabular}{|c|c|}
\hline \multicolumn{2}{|c|}{ Anisotropic diffusion performance } \\
\hline Correct classification & $2615(93.39 \%)$ \\
\hline Wrong classification & $181(6.46 \%)$ \\
\hline Not classified & $4(0.14 \%)$ \\
\hline Total & $2800(100 \%)$ \\
\hline
\end{tabular}

Table 5. Mean filtering timing

\begin{tabular}{|c|c|}
\hline \multicolumn{2}{|c|}{ Mean filter timing } \\
\hline Number of images & 2800 \\
\hline Total processing time & 8503.42 sec. \\
\hline Mean time per image & 3.034 sec. \\
\hline
\end{tabular}

Table 6. Anisotropic diffusion filtering timing

\begin{tabular}{|c|c|}
\hline \multicolumn{2}{|c|}{ Anisotropic diffusion timing } \\
\hline Number of images & 2800 \\
\hline Total processing time & 17664.5 sec. \\
\hline Mean time per image & 6.306 sec. \\
\hline
\end{tabular}

$256 \mathrm{MB}$ of RAM running Red Hat 9.0 (kernel 2.4.20-8). In tables 5 and 6 we show the running times for both filtering approaches.

\section{The Quality Measure}

The DNIC database quality is very heterogeneous, as no objective quality requirement was imposed on the acquisition process. One of the goals of this project is to define a quality measure (QI) that enables to reject bad impressions in the digitalization steps and improve the average database quality by the substitution of bad cards.

For that aim, fingerprint images are divided in blocks of $16 \times 16$ pixels. A block class is defined using Gabor filters as in [3]. We obtain eight Gabor features for each block. A block is considered to have a good quality if the standard deviation of the eight features is lower than a given threshold (420) and otherwise it is considered bad. 


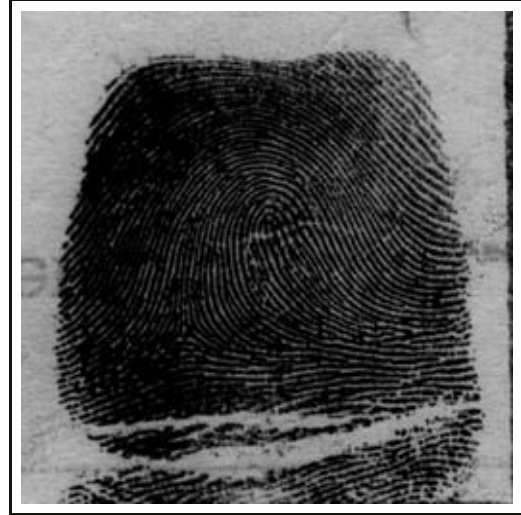

(a) Fingerprint with Bad quality $Q I_{\text {Global }}=13.289$ $Q I_{\text {Local }}=1.5873$

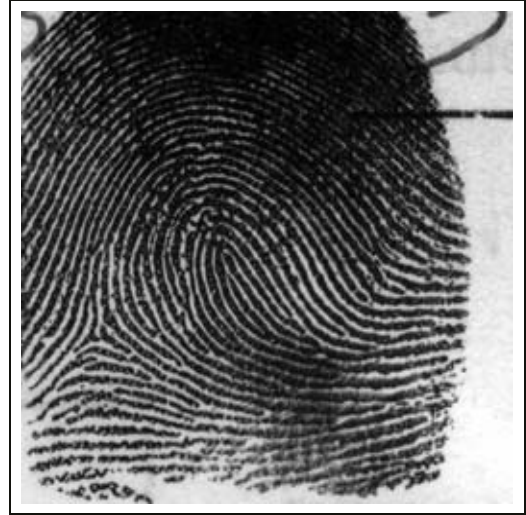

(b) Fingerprint with Poor quality $Q I_{\text {Global }}=34.03$ $Q I_{\text {Local }}=70.33$

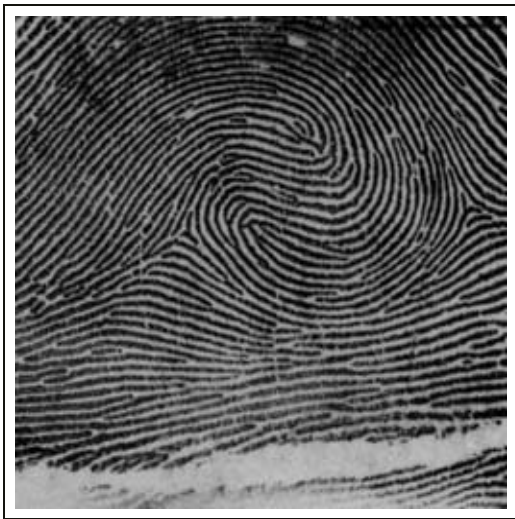

(c) Fingerprint with Good quality

$Q I_{\text {Global }}=93.78$

$Q I_{\text {Local }}=97.78$

Fig. 1. Example of fingerprints discriminated by their quality index

The fingerprint is accepted or rejected taking into account the global and local measures and also the number of iterations during the smoothing process.

We define a global QI (QIglobal) taken into account the whole fingerprint quality and a local QI (QIlocal) estimated over the singularities areas. Each iteration penalizes the measures in ten percent. A fingerprint is considered good if both QI are over their respective threshold. The QI definitions:

$$
\begin{gathered}
Q I_{\text {global }}=1-\frac{\text { number of bad quality front blocks }}{\text { number of front blocks }} \\
Q I_{\text {local }}=1-\frac{\text { number of bad quality front blocks in the mask }}{\text { number of front blocks in the mask }}
\end{gathered}
$$


The $Q I$ thresholds were learned from a training set done by the DNIC experts. A subset of fingerprints was drawn from the database. These fingerprints were analysed and categorized as good, poor or bad by the experts. This training set was used to learn the quality thresholds $\left(Q I_{\text {goodth }}=50 Q I_{\text {regularth }}=35\right)$

Perceptually the quality measure has shown to be consistent with the technicians criteria when we analyse the results over the database. We continue working looking forward this measure will work as a confidence index of the classification algorithm.

\section{Concluding Remarks}

In this work we studied and evaluated the performance improvement achieved after the modifications introduce in an automatic fingerprint classification algorithm. We defined a quality measure that has shown consistency with the technician's criteria. The modifications to the original algorithm are proposed and evaluated over images extracted from a 4 million fingerprint card archive held by DNIC.

Our future work will include improvements on the algorithm and further testing. For the anisotropic diffusion we will try working with vectors in $S^{2}$ to avoid the normalization in each step and we will also look for an automatic stopping criteria.

\section{Acknowledgments}

We want to acknowledge the DNIC staff, specially: Raúl Marcora, Jorge Millot, Graciela Nogueira and Raquel Abal.

\section{References}

1. Alberto Bartesaghi, Alicia Fernandez, and Alvaro Gomez. Performance evaluation of an automatic fingerprint classification algorithm adapted to a vucetich based classification system. In LNCS 2091, pages 259-265, 2001.

2. K. Karu and A. Jain. Fingerprint classification. Pattern Recognition, 29(3):389-404, 1996.

3. Lin Lin Shen, Alex Kot, and WaiMun Koo. Quality Measures of Fingerprint Images. School of Electrical and Electronic Engineering, Nanyang Technological University, Singapore, 2000.

4. A. Pardo and G. Sapiro. Vector Probability Diffusion. IEEE Signal Processing Letters, 8(4):106-109, April 2001.

5. P. Perona. Orientation Diffusion. IEEE Transactions on Image Processing, 7(3):457467, March 1998.

6. P. Perona and J. Malik. Scale-space and Edge Detection Using Anisotropic Diffusion. IEEE Transactions on Pattern Analysis and Machine Intelligence,12(7):629-639, July 1990.

7. N. Ratha, K. Karu, S. Chen, and A. Jain. A real-time matching system for large fingerprint databases. IEEE Pattern Analysis and Machine Intelligence, 18(8), 1996.

8. B. Tang, G. Sapiro, and V. Caselles. Diffusion of general data on non-flat manifolds via harmonic maps theory: The direction diffusion case. International Journal Computer Vision, 36(2):149-161, February 2000. 